\title{
Revisión del tratamiento y sobrevida del mesotelioma pleural maligno en el INER
}

\author{
Rufino Echegoyen-Carmona,凶 Catalina Camacho-Mendoza
}

Instituto Nacional de Enfermedades Respiratorias Ismael Cosío Villegas, Ciudad de México.

Trabajo recibido: 01-X-2014; aceptado: 10-VIII-2015

\begin{abstract}
RESUMEN. Introducción: El mesotelioma pleural aún se considera incurable, el tratamiento mejora los síntomas y prolonga la vida. El tiempo de sobrevida al diagnóstico es de 4 a 16 meses según el tipo de mesotelioma. Objetivo: Revisar el tratamiento y la sobrevida de los pacientes atendidos en el instituto. Material y métodos: Estudio retrospectivo de 90 expedientes de enero de 2006 a diciembre de 2009 con diagnóstico de mesotelioma pleural maligno. Se revisó: sexo, edad, tipo histológico, condición física, estadío de la enfermedad (UICC/AJCC), tratamiento con Cisplatino/Pemetrexed (C/P). Cisplatino/Vinorrelbina (C/V) y Cispatino/Epirrubicina (C/E), resultados (RECIST) y sobrevida global media (SGM). Resultados: Se encontraron 84 (93.3\%) mesoteliomas epitelioides y 6 (6.6\%) sarcomatosos o mixtos; 68 masculinos y 22 femeninos con ECOG de 1 en 50 (55.5\%) y estadio III y IV en 53 (58.8\%). En una primera fase del tratamiento todos recibieron 6 ciclos de quimioterapia (QT), obteniéndose $41(45.5 \%)$ de respuestas parciales, 25 (27.6\%) con progresión y 24 (26.6\%) fallecieron. En la segunda fase del tratamiento 46 pacientes recibieron una segunda línea de QT de 4 a 6 ciclos y en 20 pacientes tratamiento multimodal con QT, pleuroneumonectomía con linfadenectomía mediastinal y en 2 radioterapia. En este grupo un paciente se complicó con empiema, 1 con fístula bronquial y otro con metástasis cerebral. La relación QT/SGM muestra: C/P 15.1 meses, C/E 14.6 meses, C/V 14.3 meses. Los pacientes con tratamiento multimodal tuvieron una sobrevida mayor, uno contaba 40 meses al terminar la revisión y otro con 48 meses aún continuaba con vida. Conclusiones: La QT con C/V o C/E, dan resultados semejantes a C/P, la terapia multimodal da una sobrevida mayor.
\end{abstract}

Palabras clave: Quimioterapia, inmunohistoquímica, pleuroneumonectomía, progresión tumoral.

ABSTRACT. Introduction: It is well known that the malignant pleural mesothelioma (MPM) is refractory to treatment. The treatment only improves the symptoms and prolongs the life. The survival from the diagnosis is 4-16 months, depending on the mesothelioma type. Objective: This is a review of the treatment and the median survival time of patients that were treated at the Institute. Material and methods: A retrospective study in 90 patients who were treated from January 2006 to December 2009 was done. Sex, age, histological type, performance status (ECOG), stage (UICC/AJCC), treatment with Cisplatin/Pemetrexed (C/P). Cisplatin/Vinorelbine (C/V) y Cispatin/Epirubicin (C/E), and median overall survival (SGM) were registered. Results: 84 (93.3\%) patients had epithelioid mesothelioma and in $6(6.6 \%)$ sarcomatous or mixed mesothelioma were diagnosed; there were 68 male and 22 female, whose had ECOG 1 in 50 (55.5\%) and stage III-IV in 53 (58.8\%). At the first phase of treatment, all the patients received 6 cycles of QT and the response was evaluated as: 41 (45.5\%) had partial response, 25 $(27.6 \%)$ had progressive disease and $24(26.6 \%)$ were death. At the second treatment, 46 patients received 4 to 6 cycles of a second line of QT, and 20 patients multimodal treatment: QT, pleuro neumonectomy with mediatinal linfadenectomy and RT in two cases. In this group, one patient had empiema, one bronchial fistula and one brain matastasis. The index QT/SGM showed: C/P 15.1 months, C/E 14.6 months, C/V 14.3 months. Patients with multimodal treatment had a greater survival: one had 40 months and another one, with 48 months, when the study was finished. Conclusions: The chemotherapy with $\mathrm{C} / \mathrm{V}$ or $\mathrm{C} / \mathrm{E}$ was similar as $\mathrm{C} / \mathrm{P}$. Multimodal chemotherapy resulted in greater survival.

Key words: Chemotherapeutic, immunohistochemistry, extrapleural neumonectomy, tumor progression.

\section{INTRODUCCIÓN}

El mesotelioma es un tumor que se inicia con un engrosamiento de la pleural parietal, progresa hacia la pleura visceral, las cisuras, el pulmón, el mediastino, invade el diafragma y la cavidad peritoneal. También puede invadir los tejidos blandos del tórax. La mayor parte de los pacientes fallecen por invasión progresiva de los tejidos adyacentes o por metástasis a distancia. ${ }^{1,2} \mathrm{De}$ acuerdo con la Organización Mundial de la Salud en su clasificación más reciente, de 2004, reconoce tres variantes: el mesotelioma epitelioide, el sarcomatoide y el mixto. El diagnóstico de precisión se efectúa mediante el estudio inmunohistoquímico de una biopsia tumoral. El mesotelioma más frecuente es el epitelioide pero a la vez es el menos agresivo, con una sobrevida de 10 a 17 meses, en tanto que en el mesotelioma sarcomatoso refieren una sobrevida de 4 a 7 meses después 
del diagnóstico. En el mesotelioma mixto, la sobrevida se encuentra entre los anteriores. . $^{3,4}$

El tratamiento actual del mesotelioma pleural maligno comprende: 1) la quimioterapia (QT), 2) la radioterapia, y 3) la cirugía. En el primer grupo se incluyen los agentes químicos, los inmunológicos y los antiangiogénicos. La radioterapia tiene como inconveniente la gran extensión del tumor, por lo que se indica para radiar sitios concretos: el de la toracocentesis, la pleurocoscopia, la pleurodesis, o el mediastino. La cirugía comprende la pleurodesis, la pleurectomía y la pleuroneumonectomía. Actualmente se prefiere el tratamiento multimodal (quimioterapia, cirugía y radioterapia) en función del tipo de enfermo y la extensión del mesotelioma. A pesar de la terapéutica actual, al mesotelioma se le considera un tumor incurable, lo más que se ha podido hacer es incrementar la vida y disminuir los síntomas. ${ }^{5}$

La asociación de Pemetrexed más Cisplatino se considera como la mejor asociación quimioterápica para este padecimiento; sin embargo, se han reportado otras asociaciones con resultados semejantes.

Teniendo en cuenta el incremento del mesotelioma pleural en nuestro medio, la situación socioeconómica y cultural de nuestra población, así como el costo elevado del Pemetrexed, se pensó comparar los resultados de la asociación del Cisplatino con otros quimioterápicos más baratos que disminuyeran los abandonos frecuentemente observados en los pacientes. El presente trabajo tiene como objetivo revisar el tratamiento y la sobrevida global media de los pacientes con mesotelioma pleural en el instituto.

\section{MATERIAL Y MÉTODOS}

Se realizó un estudio retrospectivo de los expedientes con diagnóstico de mesotelioma pleural maligno comprobado con inmunohistoquímica, atendidos de enero de 2006 a diciembre de 2009, en el Instituto Nacional de Enfermedades Respiratorias (INER). La revisión comprendió 90 pacientes, se registraron los siguientes datos: edad, sexo, estudios radiográficos, tipo histológico, condición física (ECOG), y estadificación según (UICC/ AJCC). ${ }^{5} \mathrm{~A}$ todos se les dio una primera línea de quimioterapia con alguna de las siguientes asociaciones: 1 ) Cisplatino $\left(60 \mathrm{mg} / \mathrm{m}^{2}\right) /$ Pemetrexed $\left(500 \mathrm{mg} / \mathrm{m}^{2}\right)$, 2) Cisplatino $\left(60 \mathrm{mg} / \mathrm{m}^{2}\right) /$ Vinorrelbina $\left(25 \mathrm{mg} / \mathrm{m}^{2}\right)(\mathrm{CV})$ y 3$)$ Cisplatino $\left(60 \mathrm{mg} / \mathrm{m}^{2}\right) /$ Epirrubicina $\left(60 \mathrm{mg} / \mathrm{m}^{2}\right)(\mathrm{CE})$, administradas cada 21 días durante 6 ciclos. Al término de esta fase se evaluaron las respuestas de acuerdo con el criterio inicial de RECIST: estudio clínico y medición unidimensional de las lesiones blanco en la radiografía simple de tórax, y el pronóstico en los posibles quirúrgicos, evaluado con tipo histológico, estado físico, estadio del mesotelioma y biometría hemática (EORTC). En estas condiciones se formaron dos grupos: el que siguió con quimioterapia y el que continuó con tratamiento multimodal: cirugía, radioterapia y quimioterapia.

El grupo tratado con quimioterapia continuó con una segunda línea de 4 a 6 ciclos integrada con otra de las asociaciones señaladas al principio y diferente a la que recibieron inicialmente. En los que fue posible se les proporcionó una tercera línea integrada en forma semejante.

En el grupo con tratamiento multimodal se efectuó pleuroneumonectomía con linfadenectomía mediastinal, radioterapia mediastinal y una segunda y tercera línea de quimioterapia.

Se valoró la sobrevida global media (SGM), como la mediana del tiempo transcurrido desde el inicio del tratamiento hasta la última fecha en que asistieron los pacientes a consulta relacionada con las líneas quimioterapéuticas propuestas.

\section{RESULTADOS}

Tipo de mesotelioma: De los 90 pacientes estudiados, $84(93.3 \%)$ casos correspondieron al mesotelioma epitelioide y $6(6.6 \%)$ al sarcomatoso o mixto.

Edad y sexo: 68 (75.5\%) eran hombres con edad media de 59 años y 22 (24.4\%) mujeres con edad media de 48 años.

ECOG y estadificación: 50 pacientes, (55.5\%) tenían un ECOG de 1. En 53 (58.8\%) el estadio del mesotelioma se encontró entre III y IV.

\section{Primera fase del tratamiento}

Una vez confirmado el diagnóstico de mesotelioma pleural mediante estudio inmunohistoquímico, y sin selección específica, se les proporcionó una primera línea de quimioterapia con alguna de las siguientes asociaciones: 1) Cisplatino $\left(60 \mathrm{mg} / \mathrm{m}^{2}\right) /$ Pemetrexed $\left(500 \mathrm{mg} / \mathrm{m}^{2}\right)$, 2) Cisplatino $\left(60 \mathrm{mg} / \mathrm{m}^{2}\right) /$ Vinorrelbina $(25$ $\left.\mathrm{mg} / \mathrm{m}^{2}\right)(\mathrm{CV})$ y 3) Cisplatino $\left(60 \mathrm{mg} / \mathrm{m}^{2}\right) /$ Epirrubicina $\left(60 \mathrm{mg} / \mathrm{m}^{2}\right)(\mathrm{CE})$, administradas cada 21 días durante 6 ciclos (tabla 1).

Al terminar los 6 ciclos de la primera línea quimioterápica, se evaluaron las respuestas al tratamiento mediante estudio clínico, exámenes de laboratorio y radiografías simples de tórax. De acuerdo con el criterio inicial de RECIST, las mediciones fueron unidimensionales de las lesiones blanco (tabla 2).

En 4 pacientes con mesotelioma epitelioide y derrame masivo se evacuó una porción importante de líquido con objeto de mejorar la disnea, y se efectuó pleurodesis. 
Tabla 1. Quimioterapia.

\begin{tabular}{lcr}
\hline Líneas terapéuticas & Número & $\%$ \\
\hline Cisplatino/Pemetrexed & 36 & 40.0 \\
Cisplatino/Vinorrelbina & 30 & 33.3 \\
Cisplatino/Epirrubicina & 24 & 26.6 \\
Total & 90 & 100.0 \\
\hline
\end{tabular}

Tabla 2. Respuesta de acuerdo con el criterio inicial de RECIST.

\begin{tabular}{cccc}
\hline Total $(\mathrm{n})$ & $\begin{array}{c}\text { Respuesta } \\
\text { parcial }\end{array}$ & Progresión & Fallecimientos \\
\hline 90 & $41(45.5 \%)$ & $25(27.6 \%)$ & $24(26.6 \%)$ \\
\hline
\end{tabular}

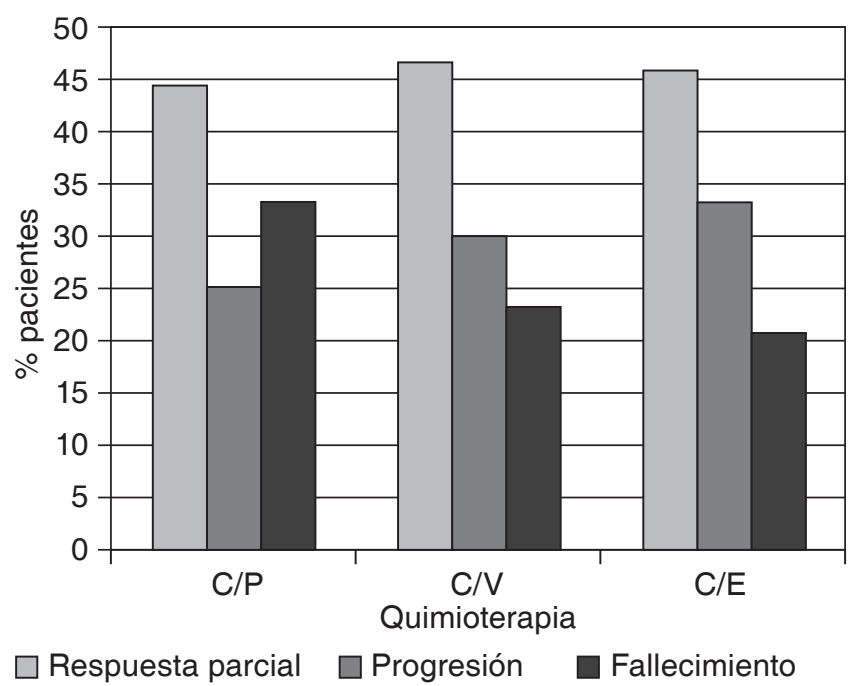

Figura 1. Respuesta a la quimioterapia de los 90 pacientes en la primera fase, con C: Cisplatino; P: Pemetrexed; V: Vinorrelbina; E: Epirrubicina.

Los resultados a cada una de las líneas quimioterápicas se muestra en la figura 1.

\section{Segunda fase del tratamiento}

Para este momento del tratamiento se tenían dos grupos:

a) Grupo tratado con quimioterapia: Comprendió 6 pacientes: a) los pacientes con progresión, b) a los que se hizo pleurodesis, c) los que rechazaron la cirugía mayor y d) aquéllos en los que esta cirugía no se pudo efectuar. Este grupo continuó con una segunda línea de 4 a 6 ciclos de quimioterapia integrada con otra de las asociaciones señaladas al

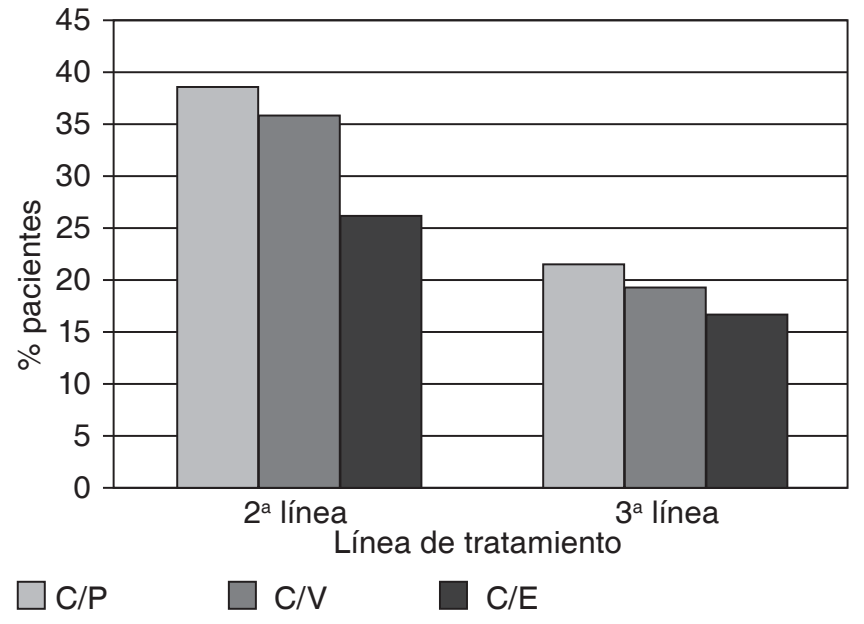

Figura 2. Segunda fase de quimioterapia en 42 pacientes, en donde C: Cisplatino; P: Pemetrexed; V: Vinorrelbina; E: Epirrubicina. El número de pacientes fue para la $2^{\mathrm{a}}$ línea: $\mathrm{C} / \mathrm{P}$ 16, C/V 15, C/E 11; y para la 3ª́nea: C/P 9, C/V 8 y C/E 7.

principio y diferente a la que recibieron inicialmente. De ellos, 24 recibieron una tercera línea (figura 2).

b) Grupo con tratamiento multimodal: Comprende 20 casos: a los 41 pacientes con respuesta parcial se les efectuó TAC de tórax y se valoró el posible pronóstico de acuerdo con la clasificación inicial de EORTC, obteniendo 19 (46.3\%) con buen pronóstico y $22(53.6 \%)$ con mal pronóstico. De los 41 enfermos se seleccionaron 34 para llevarlos a cirugía y se efectuaron los estudios necesarios para pleuroneumonectomía con linfadenectomía mediastinal. Sin embargo, sólo se pudo efectuar esta operación en 20 , porque 6 no aceptaron y en 8 no se pudo completar la operación porque el mesotelioma había progresado a mediastino, abdomen o a pared costal. Después de la cirugía se les proporcionó una segunda línea de quimioterapia de 4 a 6 ciclos con alguno de los esquemas antes señalados y posteriormente, una tercera línea con Cisplatino $(60 \mathrm{mg} /$ $\left.\mathrm{m}^{2}\right) /$ Gencitabina $\left(800 \mathrm{mg} / \mathrm{m}^{2}\right)$ o Cisplatino/Erlotinib (150 mg/día). Dos enfermos recibieron radioterapia mediastinal.

Como complicaciones quirúrgicas se encontró: un caso con infección a cavidad torácica y otro con fístula bronquial. Un paciente hizo metástasis cerebral.

SGM: Considerando la SGM como la mediana del tiempo transcurrido desde el inicio del tratamiento hasta la última fecha en que asistieron los pacientes a consulta, y relacionándolo con los esquemas quimioterapéuticos propuesto se obtuvo una SGM a los dos años, de 15.1 meses con la asociación C/P, de 14.6 meses para C/E y de 14.3 meses para C/V (tabla 3). 
Tabla 3. Sobrevida global media.

\begin{tabular}{ccc}
\hline Quimioterapia & N (\%) & SGM meses \\
\hline C/P & $36(40.0)$ & 15.1 \\
C/V & $30(33.3)$ & 14.3 \\
C/E & $24(26.6)$ & 14.6 \\
\hline
\end{tabular}

C: Cisplatino; P: Pemetrexed; V: Vinorrelbina; E: Epirrubicina.

\section{DISCUSIÓN}

Ha habido un incremento del mesotelioma pleural en el sexo femenino, este hecho se explica porque hoy en nuestro país, las mujeres participan en la manufactura de objetos con asbesto, viven en casas con techos de asbesto o localizadas en zonas industriales donde se utiliza este material. ${ }^{6-8}$ Llama la atención que $55.5 \%$ de pacientes tenga un ECOG de 1, que contrasta con el $58.8 \%$ de casos con estadios III y IV del padecimiento y también que la diferencia en la edad media y la enfermedad avanzada sea más frecuente en el sexo masculino que en el femenino. Probablemente estas situaciones se deban a que los pacientes asisten en forma tardía a consulta por falta de educación médica y que los hombres atribuyen sus molestias al trabajo, lo que impide diagnosticar en forma temprana la enfermedad.

En relación con el tipo de mesotelioma, no diferimos de otros autores, el mesotelioma epitelioide es más frecuente.

Por lo que respecta al tratamiento, la European Society for Medical Oncology en 2002 hizo una revisión sobre la terapéutica del mesotelioma pleural abordando y comentando los aspectos sobre quimioterapia, radioterapia y cirugía. Ellis et al. ${ }^{9}$ presentan una revisión del tratamiento quimioterápico en pacientes con mesotelioma pleural avanzado y concluyen que la quimioterapia con Cisplatino y Pemetrexed proporcionan mejores resultados que cualquiera de estos quimioterápicos aislados.

La American Society of Clinical Oncology ${ }^{10}$ opina que en el tratamiento del mesotelioma pleural deben utilizarse por lo menos dos quimioterápicos en pacientes con ECOG 0-1 y consideró adecuado el tratamiento con Pemetrexed $500 \mathrm{mg} / \mathrm{m}^{2}$ y Cisplatino $75 \mathrm{mg} / \mathrm{m}^{2}$ cada 3 semanas agregando un suplemento de Vit B12 1000 microgramos mensuales y ácido fólico $0.4-1.0 \mathrm{mg}$ con objeto de disminuir la toxicidad de la asociación. Ceresoli ${ }^{11}$ refiere que la asociación de Pemetrexed con Carboplatino da resultados semejantes, con menos toxicidad.

Vogelzang et al.12 consideran que la asociación de Cisplatino con Pemetrexed o Gemcitabina es el tratamiento estándar para el mesotelioma y que los dos son igualmente efectivos. El agregar Bevacizumab no incrementa substancialmente la sobrevida.

Jassem ${ }^{13}$ estudió 243 pacientes con mesotelioma avanzado divididos en dos grupos: 123 tratados con Pemetrexed y 120 con cuidados de soporte básico. Reportó una ligera disminución de la progresión del mesotelioma en los pacientes que recibieron Pemetrexed, aunque sin mejoría en la sobrevida media.

En el tratamiento del mesotelioma se han usado varias asociaciones, así tenemos que en 1996 Ong $^{10}$ refiere respuestas de mejoría del 5 al $16 \%$ asociando la Doxorrubicina y la Epirrubicina con el Cisplatino o el Carboplatino. En 1995 Planting et al. ${ }^{14}$ asociaron el Cisplatino con el Etoposido. Posteriormente, en 1997 Sahumond ${ }^{15}$ refiere este esquema en un grupo de pacientes en fase II. Catagneto et $a .^{16}$ indicaron la asociación de Cisplatino con la Gemcitabina y Steele et al. ${ }^{17}$ la asociación de Cispatino con la Vinorrelbina.

Respecto al Erlotinib, Shepherd ${ }^{18}$ en un estudio multicéntrico de 731 pacientes con NSCLC avanzado comparó un grupo tratado con Erlotinib con otro de cuidados básicos de soporte y encontró una sobrevida media mayor en los primeros. Bareschino et al. ${ }^{19}$ refieren el uso de Erlotinib como quimioterápico de primera línea en 53 pacientes con NSCLC avanzado, obtuvieron no progresión de la enfermedad en 28 $(52.8 \%)$ a las 6 semanas y una respuesta parcial en 11 casos $(20.7 \%)$.

Como podemos observar, la bibliografía sobre quimioterapia varía entre el uso de un solo medicamento, la asociación de varios y la comparación con soportes básicos. En general los autores coinciden que la asociación de Cisplatino con Pemetrexed es la mejor.

Actualmente se prefiere el tratamiento multimodal con quimioterapia, cirugía y radioterapia. Este tumor permite la disección y la separación del tejido tumoral del sano; sin embargo, es necesario tener en cuenta la imposibilidad del cirujano para eliminar restos tumorales microscópicos, causa habitual de la recurrencia tumoral. La quimioterapia adyuvante y la radioterapia postquirúrgica tienen la finalidad de resolver este aspecto, pero la indicación de la radioterapia se limita a radiar los sitios donde se piensa quedan células neoplásicas, como el hilio pulmonar, el mediastino o el diafragma.

En relación con la indicación de la pleurodesis, Tan et al. ${ }^{20}$ revisaron las indicaciones de este acto quirúrgico, el agente a utilizar y la técnica para efectuarla, así como los resultados obtenidos en los derrames pleurales malignos. Garland et al..$^{21}$ consideran que la pleurodesis, la cirugía citorreductiva y la pleuroneumonectomía tienen como objetivo el control de los síntomas, alargar el tiempo de progresión y la posible curación del mesotelioma. 
En los enfermos donde se ha efectuado el tratamiento multimodal, diferentes autores reportan una sobrevida de 4 a 5 años. De Perrot et al., ${ }^{22}$ en 2007 refiere que en 50 casos operados de neumonectomía por mesotelioma obtuvieron una sobrevida a 3 años en $24 \%$ de los casos. Algunos autores consideran que la pleuroneumonectomía es una buena opción terapéutica; sin embargo, insisten que debe tenerse en cuenta la posibilidad de recurrencias locales y metastásicas a los nodos mediastinales, ya que encontraron que la sobrevida fue superior en aquéllos que no tenían N2 metástasis. Señalan, asimismo, que la radioterapia adyuvante es útil en el control de las recurrencias locales pero concluyen que la pleuroneumonectomía en pacientes con mesotelioma y nodos metastásicos aún es motivo de controversia. ${ }^{23,24}$

Robinson et al. ${ }^{1}$ señalan que actualmente la curación del mesotelioma pleural es ocasional, ya que ningún régimen terapéutico ha probado ser curativo; aunque, el tratamiento es importante porque prolonga la vida, controla los síntomas, mejora la condición general del paciente y la calidad de vida. ${ }^{25}$

Teniendo en cuenta la posición socioeconómica y cultural de nuestra población que determinan, asistencias tardías a consulta médica, ingresos en estadios avanzados y abandonos frecuentes por problemas económicos o familiares, los resultados obtenidos en este trabajo con las asociaciones Cisplatino/Vinorrelbina o Cisplatino/Epirrubicina, son semejantes a los de Pemetrexed/Cisplatino, pero siendo más económicas las primeras, nos permiten administrarlas en forma alternativa durante tiempos más prolongados.

\section{CONCLUSIONES}

1. La quimioterapia con Cisplatino/Vinorrelbina o Cisplatino/Epirrubicina, dan resultados semejantes a los obtenidos con Pemetrexed/Cisplatino, y son más económicas.

2. Con el tratamiento multimodal, diferentes autores reportan sobrevidas de 48 a 60 meses. En nuestro caso, dos enfermos (2.22\%) sobrevivieron 40 y 48 meses, respectivamente.

3. En nuestro medio no es fácil determinar la supervivencia real. Los pacientes esperan una curación en corto tiempo, abandonan el tratamiento o buscan terapias alternativas. A largo plazo los familiares no proporcionan la información necesaria.

\section{REFERENCIAS}

1. Robinson BW, Musk AW, Lake RA. Malignant mesothelioma. Lancet 2005;366(9483):397-408.

2. Corson JM. Pathology of mesothelioma. Thorac Surg Clin 2004;14(4):447-460.
3. Churg A, Cagle PT, Roggli VL. Tumors of the serosal membranes. Atlas of tumor pathology. $4^{\text {th }}$ series, fascicle 3. Washington, DC: American Registry of Pathology; 2006.

4. Husain AN, Colby TV, Ordonez NG, Krausz T, et al. Guidelines for Pathologic Diagnosis of Malignant Mesothelioma: 2012 Update in Consensus from the International Mesothelioma Interest Group. Archives of Pathology \& Laboratory Medicine, 2012.

5. Rusch VW. A proposed new international TNM staging system for malignant pleural mesothelioma. From the International Mesothelioma Interest Group. Chest 1995;108(4):1122-1128.

6. Tsao AS, Wistuba I, Roth JA, Kindler HL. Malignant pleural mesothelioma. J Clin Oncol 2009;27(12):2081-2090. doi: 10.1200/JCO.2008.19.8523.

7. Burdorf A, Barendregt JJ, Swuste PH, Heederik DJ. Increasing incidence of mesothelioma in the future by professional exposure to asbestos in the past. Dutch J Med 1997;141:1093-1098.

8. Echegoyen CR. Epidemiología clínica del mesotelioma pleural maligno en el INER. Neumol Cir Torax 2012;71(3):219-225.

9. Ellis P, Davies AM, Evans WK, Haynes AE, Lloyd NS; Lung Cancer Disease Site Group of Cancer Care Ontario's Program in Evidence-based Care. The use or chemotherapy in patients with advanced malignant pleural mesothelioma: a systematic review and practice guideline. J Thorac Oncol 2006;1(6):591-601.

10. Ong ST, Vogelzang NJ. Chemotherapy in malignant pleural mesothelioma. A review. J Clin Oncol 1996;14(3):1007-1017.

11. Ceresoli G, Zucali P, Favaretto A, et al. Phase II study of pemetrexed plus carboplatin in malignant pleural mesothelioma. J Clin Oncol 2006;24(9):1443-1448.

12. Vogelzang NJ, Rusthoven JJ, Symanowski J, et al. Phase III study of pemetexed in combination with cisplatin versus cisplatin alone in patients with malignant pleural mesothelioma. J Clin Oncol 2003;21(14):2636-2644.

13. Jassem J, Ramlau R, Santoro A, et al. Phase III trial of pemetrexed plus best supportive care compared with best supportive care in previously treated patients with advanced malignant pleural mesothelioma. J Clin Oncol 2008;26(10):1698-1704. doi: 10.1200/JCO.2006.09.9887.

14. Planting AS, van der Burg ME, Goey SH, et al. Phase II study of a short course of weekly high-dose cisplatin combined with long-term oral etoposide in pleural mesothelioma. Ann Oncol 1995;6(6):613-615.

15. Sahmoud T, Postmus PE, van Pottelsberghe C, et al. Etoposide in malignant pleural mesothelioma; two phase II trials of the EORTC Lung Cancer Cooperative Group. Eur J Cancer 1997;33(13):2211-2215.

16. Castagneto B, Zai S, Dongiovanni D, et al. Cisplatin and gemcitabine in malignant pleural mesothelioma: a phase II study. Am J Clin Oncol 2005;28(3): 223-226.

17. Steele JP, Shamash J, Evans MT, Gower NH, Tischkowitz MD, Rudd RM. Phase // study of vinorelbine in patients with malignant pleural mesothelioma. J Clin Oncol 2000;18(23):3912-3917. 
18. Shepherd FA1, Rodrigues Pereira J, Ciuleanu T, et al.; National Cancer Institute of Canada Clinical Trials Group. Erlotinib in previously treated non small cell lung cancer. N Engl J Med 2005;353(2):123-132.

19. Bareschino MA, Schettino C, Troiani T, Martinelli E, Morgillo F, Ciardiello F. Erlotinib in cancer tratment. An Oncol 2007;18 Suppl 6:vi35-41.

20. Tan C, Sedrakyan A, Browne J, Swift S, Treasure T. The evidence on the effectiveness of management for malignant pleural effusion: a systematic review. Eur J Cardio.Thorac Surg 2006;29:829-838.

21. Garland L, Flores R, Tsao A. Individualizing mesothelioma treatment: small steps into a brighter future. Clin Lung Cancer 2010;11(6):371-383. doi: 10.3816/ CLC.2010.n.048.

22. De Perrot M, Uy K, Anraku M, et al. Impact of lymph node metastasis on outcome after extrapleural pneumonectomy for malignan pleural mesothelioma. J Thorac Cardiovasc Surg 2007;133(1):111-116.
23. Stahel R, Weder W. Neoadjuvant chemotherapy in malignant pleural mesothelioma. Lung Cancer 2005;49 Suppl 1:S69-70.

24. Weder W, Kestenholz P, Taverna C, et al. Neoadjuvant chemotherapy followed by extrapleural pneumonectomy in malignant pleural mesothelioma. J Clin Oncol 2004;22(17):3451-3457.

25. Echegoyen CR, Camacho MC. Calidad de vida y cáncer pulmonar. Neumol Cir Torax 2011;70(3):172-178.

\section{$\triangle$ Correspondencia:}

Dr. Rufino Echegoyen Carmona Instituto Nacional de Enfermedades Respiratorias Ismael Cosío Villegas. Calzada de Tlalpan Núm. 4502, Colonia Sección XVI, 14080, México, D.F. Teléfono 56664539, extensión 5292, 5133

Correo electrónico: rufino.echegoyen@gmail.com

Los autores declaran no tener conflicto de intereses. 